Limping child

\section{A limping 6 year old child with no history of illness or trauma}

\section{R G Rodrigues}

Answers on $p 182$.

A 6 year old Asian-American boy was taken to a paediatrician by his parents because they noticed that he had been limping for the past week and had begun to complain of pain in his right knee. He had never previously experienced any episodes of leg pain or limping. The patient denied a history of trauma within the previous month. The history was also negative for fever, cough, cold symptoms, rash, abdominal pain, diarrhoea, paraesthesias or numbness of the extremities, or joint pain other than the right knee.

Physical examination revealed a well appearing child. His weight was at the 25 th percentile but his height was below the fifth percentile. His vital signs were all within the normal range; he was afebrile. His right leg was not grossly swollen or deformed, no obvious lesions were seen, no point tenderness was localised, and he was able to bear weight. However, tenderness over the groin region was elicited on flexion of the hip and he was unable to fully abduct his extended right leg compared with the left leg. The range of motion at the knee and ankle were normal. An anterior drawer test and a McMurray test were negative, but a Trendelenburg test was positive. When asked to walk, the child showed a stuttering, limping gait of his right leg and complained of right knee pain. He was able to stand on the right leg alone but reported pain in the groin when asked to hop. The remainder of the examination yielded normal findings.

Results of the following laboratory tests were normal: complete blood count, serum electrolytes, calcium, magnesium, and phosphorus, erythrocyte sedimentation rate, thyroid stimulating hormone, thyroxine, and growth hormone. Rheumatoid factor and antinuclear antibody tests were negative.

Radiographs of the right knee and femur and hip (fig 1) were done and revealed a skeletal finding that pointed to the underlying disorder.

\section{QUESTIONS}

(1) What is the likely diagnosis and how does it typically present?

(2) What are alternative differential diagnoses in a child with a limp?

Postgrad Med J 2003;79:179

\section{Author's affiliations}

R G Rodrigues, Penn State Milton S Hershey Medical Center, Department of Pediatrics, H085, 500 University Drive, PO Box 850, Hershey, PA 17033, USA

Correspondence to: Dr Rodrigues; rrodrigues@psu.edu

Submitted 9 November 2002

Accepted 13 December 2002

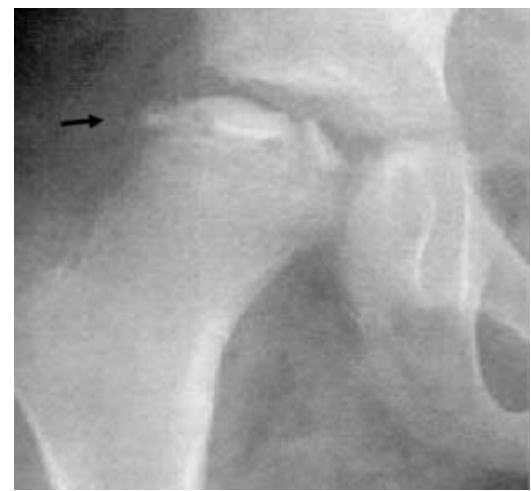

Figure 1 Radiograph of hip and femur showing necrosis of the femoral head (arrowed).

Submitted 21 September 2002

Accepted 8 November 2002

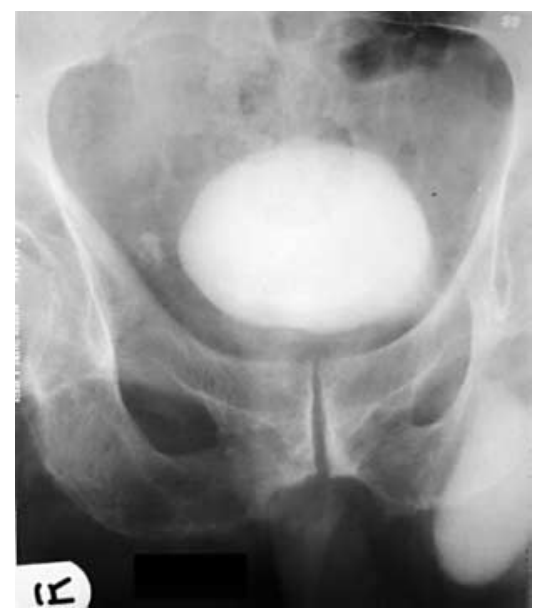

Figure 1 Intravenous urogram. 